\title{
CAPACITANCE CONTROL ON THE WIRE PRODUCTION LINE
}

\author{
Alexander Goldshtein, Galina Vavilova ${ }^{*}$, Sergey Mazikov \\ Tomsk Polytechnic University, Institute of Non-Destructive Testing, 634050 Tomsk, Russia
}

\begin{abstract}
The paper presents technical implementation of the electricalcapacitive method to perform in-process measurement of the capacitance per unit length of a single-core electric wire. The design of the electrocapacitive measuring transducer is proposed. The block diagram of the device CAP-10 developed to implement the proposed method is presented. The appearance of the device CAP-10 is showed, and its operating principle is described. It is shown that the change in water conductivity has a significant impact of the measurement result of the wire capacitance per unit length. The techniques to offset from the impact of water conductivity variation on measurement results are proposed. The technique of the device CAP-10 initial adjustment is proposed. It provides the desired function of the output signal transformation. The technique of the "operating" adjustment to correct measurement results through systematic measurement error elimination is offered.
\end{abstract}

\section{Introduction}

The capacitance per unit length is one of the main normalized values. Its stability over the entire length determines the cable products quality. It is known that the wire capacitance per unit length is largely dependent on the geometric dimensions of the wire and insulation electrical properties. Therefore, the variation of the capacitance per unit length indicates change in the wire geometric dimensions or insulation electrical properties. The need to ensure the stability of the electrical and geometrical parameters of the entire length of the cable products requires continuous in-process control of these parameters [1-5]. A continuous capacitance control can solve this problem.

The wire capacitance depends on the characteristics of in-process insulation applying; therefore, the capacitance control is to be carried out during extrusion $[1,2,6-8,10]$. The only possible embodiment of the measuring transducer in this case is the use of the tubular electrode [11] immersed in cooling water together with the test wire. This technique is widely used by the leading companies in the cable industry: Sikora (Germany), Zumbach (Switzerland) and others [12-14].

* Corresponding author:wgw@tpu.ru 


\section{The design of the measuring transducer}

Figure 1 shows the electro-capacitive measuring transducer (ECMT) used to implement the proposed method of the capacitive control. Its design is described in detail in $[9,15-17]$. The main elements of ECMT are tubular measuring electrode 1, a pair of tubular guard electrodes 2 and 3 placed in metal housing 4 and isolated from it by dielectric layer 5 . The guard electrodes are used to ensure high field uniformity in the test zone. The electrodes are connected to the alternating voltage generator 6 . The wire core and the ECMT housing are grounded. The ECMT is immersed in the cooling water bath installed in the extrusion line. The test wire 7 is permanently moved inside the ECMT.

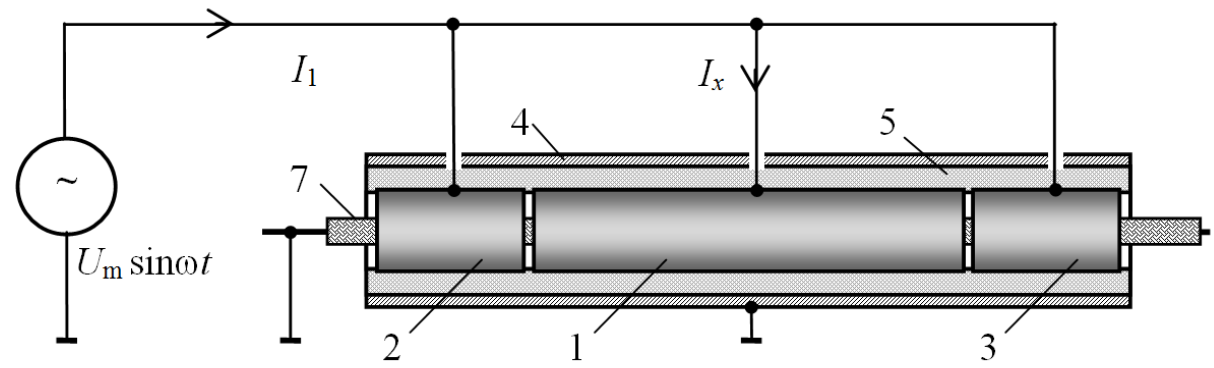

Figure 1. The ECMT design.

To minimize the dependence of the wire capacitance test result on its geometric parameters, electrical insulation properties and the change in water conductivity, it is necessary to provide an optimal ECMT design. The optimal design values for the ECMT can be selected through computer simulation using the COMSOL Multiphysics. The basic ECMT design values are the inner diameter of the tubular electrodes, the length of the measuring and guard electrodes, the distance (gap) between the measuring and guard electrodes, the inner diameter of the ECMT cylindrical housing [16].

An optimal ECMT design provides high longitudinal (axial) uniformity of the electric field in the test zone. The electric field is uniform if the values of the radial component $E_{r}$, $\mathrm{V} / \mathrm{m}$, and the electric field intensity vector $\bar{E}$ are similar, and the longitudinal component $E_{x}, \mathrm{~V} / \mathrm{m}$, (directed along the cable axis $\mathrm{x}$ ) is equal to zero at any fixed value of the radius $r$ in the measuring electrode (Figure 2).

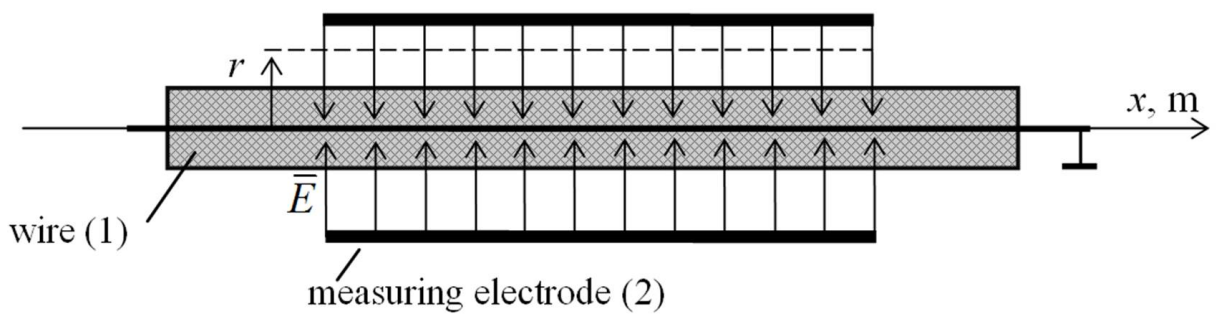

Figure 2. The field inside the transducer.

The criterion for the optimal design is the equality of the coefficient $\beta$ to one. The coefficient $\beta$ is the ratio of the capacitance per unit length in the central part of the measuring electrode $C_{l}, \mathrm{pF} / \mathrm{m}$, to the capacitance per unit length along the total length of the measuring electrode $C_{2}, \mathrm{pF} / \mathrm{m}$, (in the central part of the measuring electrode, the electric field is known to be uniform): 


$$
\beta=\frac{C_{2}}{C_{1}}
$$

The results of computer simulation show that high uniformity of the electric field inside the test zone is provided by the guard electrodes introduced into the ECMT design. In this case, the electric field spreads to the distant edges of the guard electrodes.

Figure 3 shows the distribution of the electric potentials for interaction of the ECMT electric field with the test wire plotted in equipotential lines for the case of guard electrodes of $R$ radius (equal to the inner radius of the measuring electrode), of $1 R$ length and $1 \mathrm{~mm}$ distance (gap) between the measuring electrode and the guard electrode [17].

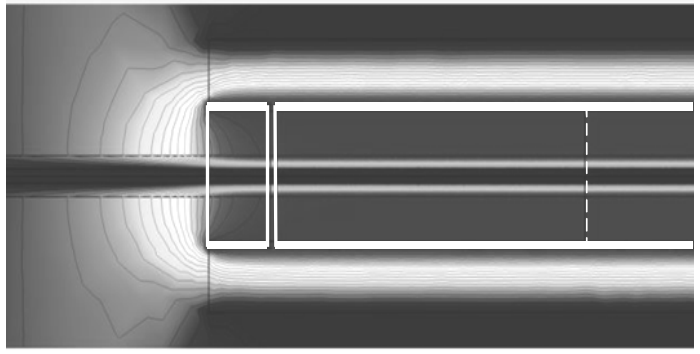

Figure 3. The electric field created by the ECMT with guard electrodes.

The basic design parameters of the optimal ECMT chosen through computer simulation [16] are as follows:

- the length of the measuring electrode is $(200 \ldots 300) \mathrm{mm}$;

- the length of the guard electrodes is $(0.5 \ldots 1) R$;

- the inner radius of the electrodes is $R_{e l} \geq 2 r$;

- the inner diameter of the housing is $R_{h} \geq 2 R_{e l}$;

- the gap between the measuring electrode and the guard electrode is (1...3) mm, where $r$ is the radius of the outer wire insulation;

$R$ is the inner radius of the measuring electrode.

\section{The device CAP-10 for measuring the wire capacitance per unit length}

The developed ECMT is the main element of the device CAP-10 which is intended for inprocess control of the capacitance per unit length for single-core electric wires.

The main technical characteristics of the device CAP-10 are as follows:

- the test object is a single-core insulated wire;

- the range of the measured capacitance per unit length is $(50 \ldots 500) \mathrm{pF} / \mathrm{m}$;

- the outer diameter of the wire insulation varies from $0.5 \mathrm{~mm}$ to $12 \mathrm{~mm}$;

- the maximum permissible measurement error for the capacitance per unit length is $2.5 \%$ of its nominal value within the range of the change in water conductivity equivalent to the variation in the $\mathrm{NaCl}$ concentration range $(0 \ldots 4) \mathrm{g} / \mathrm{l}$.

The appearance of the device CAP-10 is presented in Figure 4 and its block diagram is shown in Figure 5.

The value of test wire capacitance in ECMT 1 is transformed into the current value $I_{x}$. This signal is applied to input analogue conversion unit 2, which includes current transformer (CT) 3, current-to-voltage converter (CVC) 4 and amplitude-phase detector (APD) 5. CT is used for galvanic isolation of the electrode measuring circuit and the signal analogue conversion circuit. The CT output current $I_{x}$ is applied to CVC, where it is 
converted into voltage, and then to the APD input, in which the real and imaginary parts of the signal measurement information are differentiated $[1,9,15]$.

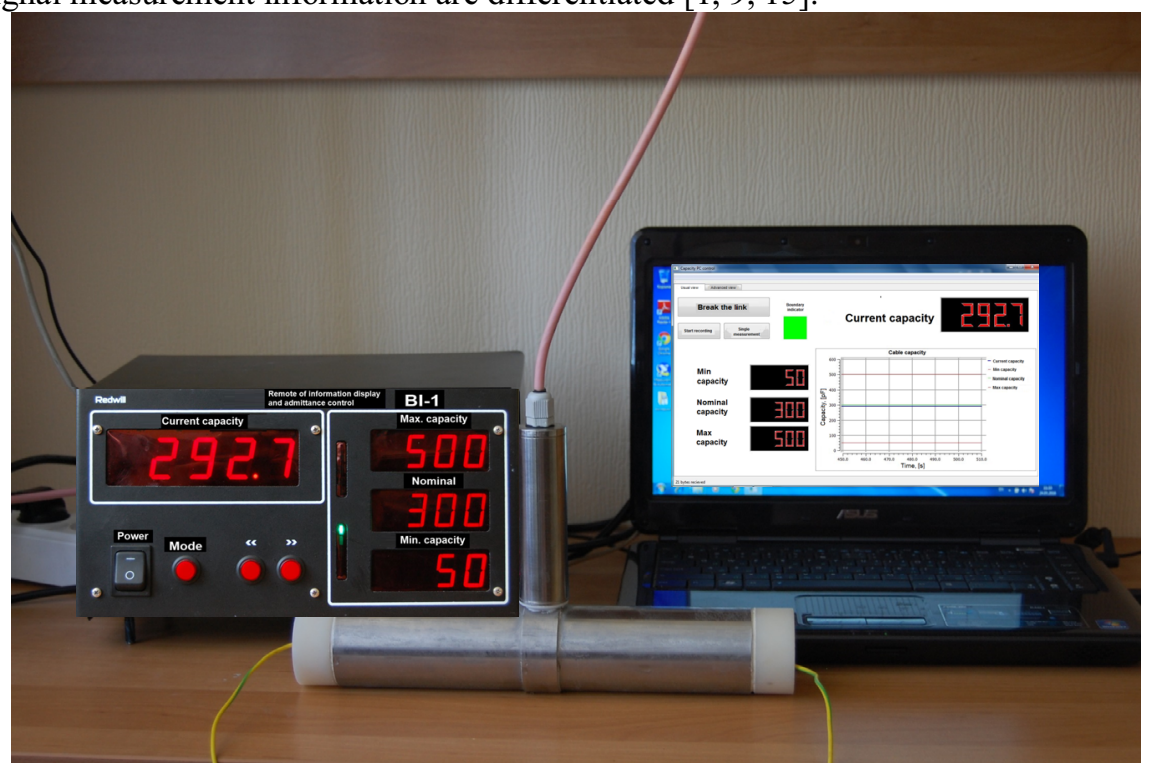

Figure 4. The appearance the device CAP-10.

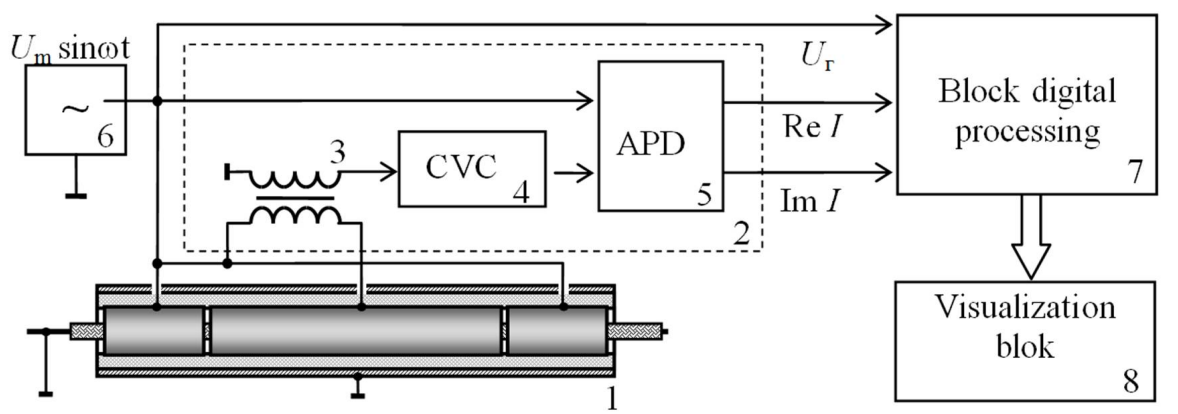

Figure 5. The block diagram of CAP-10.

The APD reference signal is the voltage of generator 6 . The APD output voltages $(\operatorname{Re} U$ и $\operatorname{Im} U$ ) proportional to the amplitude values of the complex parts of the current in the measuring electrode circuit are applied to digital processing unit 7 based on the microcontroller. Digital processing unit 7 performs filtering, measurement data averaging within 1 second and digital processing.

Visualization block unit 9 provides display of the measurement results in the form convenient for an operator. Information on the measured parameters is displayed on the panel BI-1 presented in Figure 6, $a$.

A personal computer is used to record an array of data on capacitance per unit length within the set time interval and to display the measured data. The virtual front panel of the device CAP-10 is showed in Figure 6, $b$. 


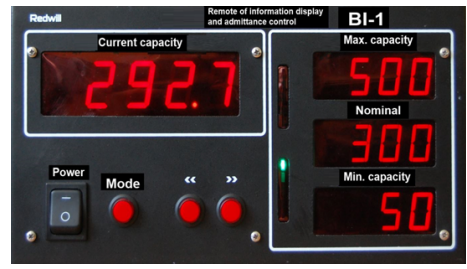

a)

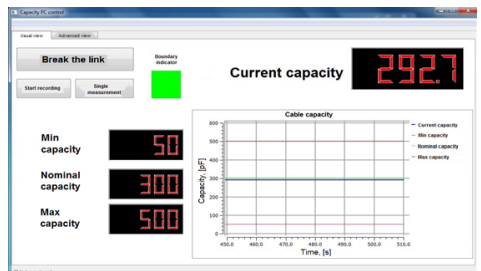

b)

Figure 6. Visualization of measured data by the device CAP-10.

\section{Detection of local defects using CAP-10}

The primary function of the wire capacitance control by CAP-10 is continuous monitoring of local defects (a cracks, a voids in a insulation etc.) in wire insulation.

Local defects in wire insulation cause sharp abrupt change in the wire capacitance as a result of sharp change in geometric dimensions and electrical insulation. The device CAP10 records the change in the capacitance. When it detects a defect, the parameters are displayed (Figure 7). The presented capability of the device CAP-10 extends the scope of its application and provides additional capabilities of spark testing in non-destructive testing in the cable industry $[1,2]$.

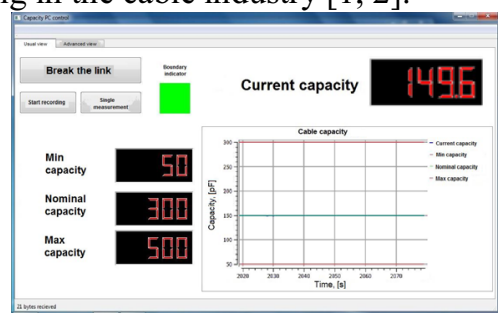

no defect

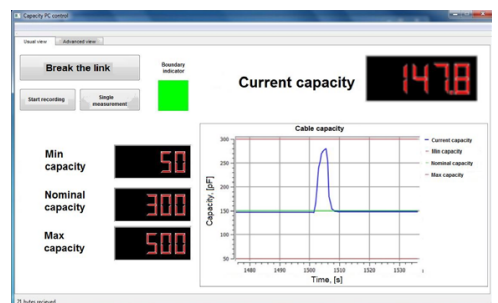

a defect is detected

Figure 7. Test results for the sample free of defects and that with a detected defect.

\section{The techniques to offset from the impact of change in water conductivity}

The algorithm for the ECMT output signal transformation in the capacitance is based on the linear function of the measured current $I_{x}, \mathrm{~mA}$, transformation into capacitance per unit length $C_{\mathrm{w}}, \mathrm{pF} / \mathrm{m}$ :

$$
C_{w}=C_{0}+k \cdot I_{x}
$$

where $C_{0}, \mathrm{pF} / \mathrm{m}$, is a constant component, $k, \mathrm{pF} /(\mathrm{m} \cdot \mathrm{A})$, is a proportionality factor. These parameters depend on the ECMT design parameters.

The studies $[1,9,15,17]$ indicate that the change in water conductivity due to change in water temperature and concentration of impurities have a significant impact on the result of the wire capacitance test. Consequently, the coefficients $\mathrm{C} 0, \mathrm{pF} / \mathrm{m}$, and $\mathrm{k}, \mathrm{pF} /(\mathrm{m} \cdot \mathrm{A})$, are the functions of the current water conductivity values. The wire capacitance test without consideration of the current water conductivity causes a measurement error that can reach $20 \%$.

The decreased impact of the change in water conductivity is implemented through the offsetting techniques based on indirect measurements of the current water conductivity. To perform the offset, the phase of the current of the measuring electrode equal to 
$t=\operatorname{Re} \dot{I}_{x} / \operatorname{Im} \dot{I}_{x}$ is determined $[9,15]$ and the generator current is measured (Figure 1) [18].

The values $C_{0}, \mathrm{pF} / \mathrm{m}$, and $k, \mathrm{pF} /(\mathrm{m} \cdot \mathrm{A})$, in functions (2) are replaced with their respective functions $C_{0}(t)$ and $k(t)$ or $C_{0}(I)$ and $k(I)$ :

$$
C_{\mathrm{w}}=C_{0}(t)+k(t) \cdot I_{x} .
$$

or

$$
C_{\mathrm{w}}=C_{0}\left(I_{1}\right)+k\left(I_{1}\right) \cdot I_{x}
$$

The functions $C_{0}(t), k(t), C_{0}\left(I_{1}\right)$ and $k\left(I_{1}\right)$ are described by the second order polynomials. The coefficients of these polynomials are determined by the design ECMT parameters.

The device CAP-10 implements both techniques to offset from the impact of change in water conductivity. The main of the techniques is based on determination of the measuring electrode current phase.

The techniques based on determination of the current generator $I_{1}$ should be used only when the phase of the measured current change not only due to change in water conductivity, but also because of the change in the ratio of the wire electric parameters $C_{x}$, $\mathrm{pF} / \mathrm{m}$, and $R_{x}, \mathrm{kOm} / \mathrm{m}$.

These techniques to offset can be used to perform an individual control of wire parameters. Switching between the two techniques is performed automatically.

\section{Metrological assurance of CAP-10}

To ensure compliance with the characterictics provided in the CAP-10 technical documentation, the device is adjusted. For this purpose, specially prepared segments of the tested wire with different insulation materials and known values of the capacitance per unit length within the limit of CAP-10 measurement are used [2, 4]. The actual values of the wire capacitance per unit length were determined in compliance with GOST 27893-88 "Communication Cables. Methods of Tests" [19].

At the manufacturing stage, the device CAP-10 is initially adjusted, i.e. the coefficients of the function of the ECMT output signal transformation into capacitance per unit length (2) are found for each implementation of the offsetting techniques. The Initial adjustment is carried out in normal weather conditions, according to GOST 2789388 [19].

If the device CAP-10 installed in the wire production line (for the first time or after its repair) exhibits excessive capacitance measurement error $(2.5 \%)$, the obtained result can be adjusted. The "operating" adjustment is automated. This adjustment is required to eliminate the additive and a multiplicative components of the systematic error. During "operating" adjustment, the device CAP-10 is placed in the cooling bath installed in the wire production line, in which the manufactured wire is placed after its extrusion.

The task of the "operating" adjustment is to determine the capacitance $\Delta C, \mathrm{pF} / \mathrm{m}$. This value is calculated as follows:

$$
\Delta C=\Delta C_{1}+\frac{\Delta C_{2}-\Delta C_{1}}{C_{x 2}-C_{x 1}}\left(C_{x}-C_{x 1}\right) .
$$

The corrected value of the capacitance per length for each wire sample is calculated as follows:

$$
C_{x}^{*}=C_{x}-\Delta C
$$

The quality of the "operating" adjustment is estimated using a test wire segment with known valid capacitance. 


\section{Conclusions}

The developed ECMT design allows selection of optimum design parameters to provide a minimum methodical measurement error caused by the influence of the wire geometric dimensions, electrical properties of insulation and change in water conductivity.

The techniques to offset from the impact of change in water conductivity and their algorithms are proposed and implemented based on determination of the measuring electrode current phase and the generator current. These techniques reduce the measurement error of the wire capacitance per unit length in the range from 50 to $500 \mathrm{pF} / \mathrm{m}$ in conditions of significant change in the water conductivity.

The feasibility of the device CAP-10 to detect local defects (a cracks, a voids in a insulation and others) in the wire insulation is shown. It expands the scope of the spark method of cable product control.

The technique of the device CAP-10 initial adjustment to provide the desired function of the output signal transformation is offered. The technique of the device CAP-10 "operating" adjustment to correct measurement results through systematic measurement error elimination is proposed.

\section{References}

[1] N.S. Starikova, V.V. Redko, G.V. Vavilova, J. Phys.: Conf. Ser. 671, 012056 (2016) doi: 10.1088/1742-6596/671/1/012056

[2] E.M. Fedorov, I.D. Bortnikov, Tech. Phys. 60, 1689 (2015) doi: $10.1134 / \mathrm{S} 1063784215110110$

[3] A.P. Surzhikov, V.V. Peshev, A.M. Pritulov, S.A. Gyngazov, Russ Phys J. 42, 490 (1999)

[4] O.V. Galtseva, S.V. Bordunov, S.N. Torgaev, IOP Conf. Ser.: Mater. Sci. Eng. 110, 012094 (2016) doi: 10.1088/1757-899X/110/1/012094

[5] D.D. Burleigh, M.V. Kuimova, Ponte 72 , 2 (2016)

[6] R. Kodermyatov, M. Ivanov, M. Yuzhakov, V. Kuznetsov, M. Yuzhakova, E. Timofeeva, MATEC Web of Conferences 48, 05004 (2016) doi: $10.1051 /$ confmatec/20164805004

[7] A. I. Nizhegorodov, A.N. Gavrilin, B. B. Moyzes, J. Phys.: Conf. Ser. 671, 012037 (2016) DOI: 10.1088/1742-6596/671/1/012037

[8] A.A. Bespalko, L.V. Yavorovich, P.I. Fedotov, Russ. J. Nondestr. Test. 47, 680 (2011) doi: 10.1134/S1061830911100068

[9] A.E. Goldshtein, G.V. Vavilova, Int. J. Syst. Appl., Eng. \& Dev, 10, 217 (2016)

[10] A.P. Surzhikov, E.N. Lysenko, V.A. Vlasov, A.V. Malyshev, E.V. Nikolaev, RUSS PHYS J. 56 (6), 681 (2013) doi: 10.1007/s11182-013-0085-6

[11] P. Fleming, L.R. Coleman, A system for monitoring fluctuations in the thickness of a cable insulating sheath GB Patent 2358928 (2001)

[12] CAPACITANCE 2000: Capacitance measuring devices for cable production lines. URL: http://sikora.net/wp-content/uploads/2016/03/CAPACITANCE_2000_GB.pdf (2016)

[13] CAPAC®/FFT: Capacitance measurement systems for cable production lines. URL: http://www.zumbach.com/pdf/Literature/CAPAC/CAPAC_Familie_CAPA.002.000 2.E.pdf (2016)

[14] W. Blohm, Proceedings Intl. IWMA Conf. Economical Processing of Rod to Wire \& Cable, 67 (1999)

[15] A.E. Goldshtein, G.V. Vavilova, S.V. Mazikov, J. Phys.: Conf. Ser. 671, 012062 (2016) doi: 10.1088/1742-6596/671/1/012062 
[16] P. Fleming, L.R. Coleman, Capacitance monitoring systems, US Patent 20030128038 (2003)

[17] A.E. Goldshtein, G.V. Vavilova, V.Yu. Belyankov, Russ. J. Nondestr. Test. 51, 86 (2015) doi: 10.1134/S1061830915020047

[18] A.E. Goldshtein, G.V. Vavilova, V.V. Redko, Method of measurement capacitance of a solid strand electrical wire RU Patent 2578658 (2016)

[19] GOST 27893-88 Telephone cables, Test methods (Standartinform, Moscow, 2010) 